Nonlinear Analysis and Differential Equations, Vol. 2, 2014, no. 3, 135 - 144

HIKARI Ltd, www.m-hikari.com

http://dx.doi.org/10.12988/nade.2014.468

\title{
A Note on Euler-Maruyama's Approximate Solutions of Stochastic Differential Equations
}

\author{
Wenfei Xu \\ College of Mathematics and Information Science \\ Henan Normal University, Henan Province, 453007, China \\ Shanshan Chen \\ College of Mathematics and Information Science \\ Henan Normal University, Henan Province, 453007, China
}

Copyright (c) 2014 Wenfei Xu and Shanshan Chen. This is an open access article distributed under the Creative Commons Attribution License, which permits unrestricted use, distribution, and reproduction in any medium, provided the original work is properly cited.

\begin{abstract}
In this paper, we focus on Euler-Maruyama's approximate solutions. Thus, some conclusions about the $p$-th $(p>1)$ moments of the errors between the unique solution $x(t)$ of the stochastic differential equation and Euler-Maruyama's approximate solutions will be established.
\end{abstract}

Mathematics Subject Classification: 60H10

Keywords: Stochastic differential equation, Euler-Maruyama's approximate solutions

\section{Introduction}

In a bit to understand the unique solution $x(t)$ of the stochastic differential equations, we often draw support from approximate solutions. Recently there exist two famous approximate solutions-Caratheodory's approximate solutions and Euler-Maruyama's approximate solutions. In this paper, we decide to discuss Euler-Maruyama's approximate solutions. 
Firstly, we will give some preconditions. Let $(\Omega, \mathrm{F}, P)$ be a complete probability space with a filtration $\left\{\mathrm{F}_{t}\right\}_{t \geq 0}$ satisfying the usual conditions. Throughout this paper, unless otherwise specified, we let $B(t)=\left(B_{1}(t), B_{2}(t), \cdots, B_{m}(t)\right)$, $t \geq 0$ be $m$-dimensional Brownian motion defined on the space. Let $0 \leq t_{0}<$ $T<\infty$. Let $x_{0}$ be an $\mathrm{F}_{t_{0}}$-measurable $R^{d}$-valued random variable such that $\mathbb{E}\left|x_{0}\right|^{2}<\infty$. Let $f: R^{d} \times\left[t_{0}, T\right] \rightarrow R^{d}$ and $g: R^{d} \times\left[t_{0}, T\right] \rightarrow R^{d \times m}$ be both Borel measurable. Now consider the $d$-dimensional stochastic differential equation of Itô type

$$
d x(t)=f(x(t), t) d t+g(x(t), t) d B(t) \text { on } t_{0} \leq t \leq T
$$

with initial value $x\left(t_{0}\right)=x_{0}$. Of course, in order to make contents in this paper meaningful, we will list two important conditions which ensure the existence and uniqueness of the solution to equation (1). They are Lipschitz and Linear growth conditions.

There exists two positive constants $\bar{K}, K$ such that

1. (Lipschitz condition) for all $x, y \in R^{d}$ and $t \in\left[t_{0}, T\right]$

$$
|f(x, t)-f(y, t)|^{2} \vee|g(x, t)-g(y, t)|^{2} \leq \bar{K}|x-y|^{2},
$$

2. (Linear growth condition) for all $(x, t) \in R^{d} \times\left[t_{0}, T\right]$

$$
|f(x, t)|^{2} \vee|g(x, t)|^{2} \leq K\left(1+|x|^{2}\right)
$$

We know that the solutions of stochastic differential equations does not often have an explicit expressions except the linear case. Thus, we often seek the approximate solution rather than the accurate solution. One of the methods we have used is Picard iteration procedure. Let us recall the Picard iterations to equation (1). Set $x\left(t_{0}\right) \equiv x_{0}$ and for $1,2, \cdots$, define

$$
x_{n}(t)=x_{0}+\int_{t_{0}}^{t} f\left(x_{n-1}(s), s\right) d s+\int_{t_{0}}^{t} g\left(x_{n-1}(s), s\right) d B(s)
$$

for $t \in\left[t_{0}, T\right]$. It is no doubt that the Picard iteration procedure is used to establish the theorem on the existence and uniqueness of the solution. However, the disadvantage of the Picard approximation is that one needs to compute $x_{0}(t), x_{1}(t), \cdots, x_{n-1}(t)$ in order to compute $x_{n}(t)$, and this will involve a lot of calculations on stochastic integrals. Therefore, we usually look for another effective method, such as Euler-Maruyama approximation procedure.

Now let us illustrate the definition of Euler-Maruyama approximate solutions. They are defined as follows: For every integer $n \geq 1$, define $x_{n}(t)=x_{0}$, 
and then for $t_{0}+(k-1) / n<t \leq\left(t_{0}+k / n\right) \wedge T, k=1,2, \cdots$

$$
\begin{aligned}
x_{n}(t) & =x_{n}\left(t_{0}+\frac{k-1}{n}\right)+\int_{t_{0}+(k-1) / n}^{t} f\left(x_{n}\left(t_{0}+\frac{k-1}{n}\right), s\right) d s \\
& +\int_{t_{0}+(k-1) / n}^{t} g\left(x_{n}\left(t_{0}+\frac{k-1}{n}\right), s\right) d B(s) .
\end{aligned}
$$

Note that if define

$$
\hat{x}_{n}(t)=x_{0} I_{\left\{t_{0}\right\}}(t)+\sum_{k \geq 1} x_{n}\left(t_{0}+\frac{k-1}{n}\right) I_{\left(t_{0}+(k-1) / n, t_{0}+k / n\right]}(t),
$$

for $t_{0} \leq t \leq T$. Then from the equations (5) and (6), we can obtain the expression of Euler-Maruyama's approximate solutions as follows:

$$
x_{n}(t)=x_{0}+\int_{t_{0}}^{t} f\left(\hat{x}_{n}(s), s\right) d s+\int_{t_{0}}^{t} g\left(\hat{x}_{n}(s), s\right) d B(s) .
$$

For the Euler-Maruyama's approximate solutions, the following results are well-known.

Theorem 1.1 [1] Under the linear growth condition (3), for all $n \geq 1$, we have

$$
\sup _{t_{0} \leq t \leq T} \mathbb{E}\left|x_{n}(t)\right|^{2} \leq C_{1}:=\left(1+3 \mathbb{E}\left|x_{0}\right|^{2}\right) e^{3 K\left(T-t_{0}\right)\left(T-t_{0}+1\right)}
$$

and for $t_{0} \leq s<t \leq T$ with $t-s \leq 1$,

$$
\mathbb{E}\left|x_{n}(t)-x_{n}(s)\right|^{2} \leq C_{2}(t-s),
$$

where $C_{2}=4 K\left(1+C_{1}\right)$. Moreover, assume that the Lipschitz condition (2) and the linear growth condition (3) hold. Let $x(t)$ be the unique solution of equation (1) and $x_{n}(t)$ be the Euler-Maruyama approximate solutions. Then, for $n \geq 1$,

$$
\mathbb{E}\left(\sup _{t_{0} \leq t \leq T}\left|x_{n}(t)-x(t)\right|^{2}\right) \leq \frac{C_{3}}{n}
$$

where $C_{3}=4 C_{2} \bar{K}\left(T-t_{0}\right)\left(T-t_{0}+4\right) \exp \left[4 \bar{K}\left(T-t_{0}\right)\left(T-t_{0}+4\right)\right]$.

Based on the above works, the aims of this paper is to discuss the $p$-th $(p>1)$ moments of the errors between the unique solution $x(t)$ of equation (1) and Euler-Maruyama approximate solutions. 


\section{Main Results}

Before giving our main result, let us state some notations.

- $\beta=\frac{1}{6}(18 K)^{\frac{p}{2}}\left(T-t_{0}\right)^{\frac{p-2}{2}}\left[\left(T-t_{0}\right)^{\frac{p}{2}}+\left(\frac{p(p-1)}{2}\right)^{\frac{p}{2}}\right]$

- $C_{1, p}:=\left(1+3^{p-1} \mathbb{E}\left|x_{0}\right|^{p}\right) e^{\beta\left(T-t_{0}\right)}$;

- $C_{2, p}=\frac{1}{2}(8 K)^{\frac{p}{2}}\left(\frac{p(p-1)}{2}\right)^{\frac{p}{2}}\left(1+C_{1, p}\right)$;

- $C_{3, p}=2^{2 p-2} \bar{K}^{\frac{p}{2}}\left(T-t_{0}\right)^{\frac{p}{2}}\left[\left(T-t_{0}\right)^{\frac{p}{2}}+\left(\frac{p^{3}}{2 p-2}\right)^{\frac{p}{2}}\right] C_{2, p} e^{2^{2 p-2} \bar{K}^{\frac{p}{2}}\left(T-t_{0}\right)^{\frac{p}{2}}\left[\left(T-t_{0}\right)^{\frac{p}{2}}+\left(\frac{p^{3}}{2 p-2}\right)^{\frac{p}{2}}\right]}$;

- $\bar{C}_{1, p}:=\left(C_{1}\right)^{\frac{p}{2}}$

- $\bar{C}_{2, p}=2^{p-1} K^{\frac{p}{2}}\left[1+\bar{C}_{1, p}+\left(1+C_{1}\right)^{\frac{p}{2}}\right]$;

- $\bar{C}_{3, p}=2^{2 p-1} \bar{K}^{\frac{p}{2}}\left(T-t_{0}\right)^{\frac{p}{2}}\left[\left(\frac{16 C_{2}}{p}\right)^{\frac{p}{2}}+\left(\frac{16 C_{2}}{p}\right)^{\frac{p}{2}}+\frac{\bar{C}_{2, p}}{2}\left(T-t_{0}\right)^{\frac{p}{2}}\right] e^{2^{2 p-2} \bar{K}^{\frac{p}{2}}\left(T-t_{0}\right)^{p}}$.

Now we can state the main results of the Euler-Maruyama's approximate solutions.

Theorem 2.1 Assume that the Lipschitz condition (2) and the linear growth condition (3) hold. Let $x(t)$ be the unique solution of equation (1) with the initial value $x\left(t_{0}\right)=x_{0} \in L^{p}\left(\Omega, R^{d}\right)$. Then, for $n \geq 1$ and $p \geq 2$,

$$
\mathbb{E}\left(\sup _{t_{0} \leq t \leq T}\left|x_{n}(t)-x(t)\right|^{p}\right) \leq \frac{C_{3, p}}{n^{\frac{p}{2}}} .
$$

Theorem 2.2 Assume that the Lipschitz condition (2) and the linear growth condition (3) hold. Let $x(t)$ be the unique solution of equation (1) with the initial value $x\left(t_{0}\right)=x_{0} \in L^{2}\left(\Omega, R^{d}\right)$. Then, for $n \geq 1$ and $1<p<2$,

$$
\mathbb{E}\left(\sup _{t_{0} \leq t \leq T}\left|x_{n}(t)-x(t)\right|^{p}\right) \leq \frac{\bar{C}_{3 p}}{n^{\frac{p}{2}}}
$$

\subsection{Some Lemmas}

Before giving the proofs of main theorems, we need to show the following some lemmas.

Lemma 2.3 Under the linear growth condition (3), for all $n \geq 1$ and $p \geq 2$,

$$
\sup _{t_{0} \leq t \leq T} \mathbb{E}\left|x_{n}(t)\right|^{p} \leq C_{1, p}
$$


Proof Fix $n \geq 1$ and $p \geq 2$ arbitrarily. Note from equation (7) that for $t_{0} \leq t \leq T$

$$
\begin{aligned}
\left|x_{n}(t)\right|^{p} & =\left|x_{0}+\int_{t_{0}}^{t} f\left(\hat{x}_{n}(s), s\right) d s+\int_{t_{0}}^{t} g\left(\hat{x}_{n}(s), s\right) d B(s)\right|^{p} \\
& \leq 3^{p-1}\left|x_{0}\right|^{p}+3^{p-1}\left|\int_{t_{0}}^{t} f\left(\hat{x}_{n}(s), s\right) d s\right|^{p}+3^{p-1}\left|\int_{t_{0}}^{t} g\left(\hat{x}_{n}(s), s\right) d B(s)\right|^{p} .
\end{aligned}
$$

Using the Hölder inequality, the martingale inequality [2, Theorem 7.2 in Section 1.7] and the definition of Euler-Maruyama approximate solutions, we can then derive that

$$
\begin{aligned}
\mathbb{E}\left|x_{n}(t)\right|^{p} & \leq 3^{p-1} \mathbb{E}\left|x_{0}\right|^{p}+2^{\frac{p-2}{2}}\left[3\left(t-t_{0}\right)\right]^{p-1} K^{\frac{p}{2}} \int_{t_{0}}^{t}\left(1+\mathbb{E}\left|\hat{x}_{n}(s)\right|^{p}\right) d s \\
& +2^{\frac{p-2}{2}} 3^{p-1}\left(\frac{p(p-1)}{2}\right)^{\frac{p}{2}}\left(t-t_{0}\right)^{\frac{p-2}{2}} K^{\frac{p}{2}} \int_{t_{0}}^{t}\left(1+\mathbb{E}\left|\hat{x}_{n}(s)\right|^{p}\right) d s \\
& \leq 3^{p-1} \mathbb{E}\left|x_{0}\right|^{p}+\beta \int_{t_{0}}^{t}\left(1+\mathbb{E}\left|\hat{x}_{n}(s)\right|^{p}\right) d s \\
& \leq 3^{p-1} \mathbb{E}\left|x_{0}\right|^{p}+\beta \int_{t_{0}}^{t}\left(1+\sup _{t_{0} \leq r \leq s} \mathbb{E}\left|x_{n}(r)\right|^{p}\right) d s
\end{aligned}
$$

for all $t_{0} \leq t \leq T$, where $\beta=\frac{1}{6}(18 K)^{\frac{p}{2}}\left(T-t_{0}\right)^{\frac{p-2}{2}}\left[\left(T-t_{0}\right)^{\frac{p}{2}}+\left(\frac{p(p-1)}{2}\right)^{\frac{p}{2}}\right]$. Consequently,

$$
\sup _{t_{0} \leq t \leq T} \mathbb{E}\left|x_{n}(t)\right|^{p} \leq 3^{p-1} \mathbb{E}\left|x_{0}\right|^{p}+\beta \int_{t_{0}}^{T}\left(1+\sup _{t_{0} \leq r \leq s} \mathbb{E}\left|x_{n}(r)\right|^{p}\right) d s .
$$

Since Gronwall inequality implies

$$
1+\sup _{t_{0} \leq t \leq T} \mathbb{E}\left|x_{n}(t)\right|^{p} \leq\left(1+3^{p-1} \mathbb{E}\left|x_{0}\right|^{p}\right) e^{\beta\left(T-t_{0}\right)},
$$

we can get

$$
\sup _{t_{0} \leq t \leq T} \mathbb{E}\left|x_{n}(t)\right|^{p} \leq\left(1+3^{p-1} \mathbb{E}\left|x_{0}\right|^{p}\right) e^{\beta\left(T-t_{0}\right)}
$$

Lemma 2.4 Under the linear growth condition (3), for all $n \geq 1$ and $t_{0} \leq$ $s<t \leq T$ with $t-s \leq 1$ and $p \geq 2$,

$$
\mathbb{E}\left|x_{n}(t)-x_{n}(s)\right|^{p} \leq C_{2, p}(t-s)^{\frac{p}{2}} .
$$


Proof Note that

$$
x_{n}(t)-x_{n}(s)=\int_{s}^{t} f\left(\hat{x}_{n}(r), r\right) d r+\int_{s}^{t} g\left(\hat{x}_{n}(r), r\right) d B(r) .
$$

Hence, we can know that easily,

$$
\begin{aligned}
& \mathbb{E}\left|x_{n}(t)-x_{n}(s)\right|^{p} \\
\leq & \gamma \mathbb{E} \int_{s}^{t}\left[1+\left|\hat{x}_{n}(r)\right|^{2}\right]^{\frac{p}{2}} d r \leq 2^{\frac{p-2}{2}} \gamma \int_{s}^{t}\left[1+\mathbb{E}\left|\hat{x}_{n}(r)\right|^{p}\right] d r \\
\leq & 2^{\frac{p-2}{2}} \gamma \int_{s}^{t}\left[1+\sup _{s \leq u \leq r} \mathbb{E}\left|x_{n}(u)\right|^{p}\right] d r \leq 2^{\frac{p-2}{2}} \gamma\left(1+C_{1, p}\right)(t-s) \\
\leq & \frac{1}{2}(8 K)^{\frac{p}{2}}\left(\frac{p(p-1)}{2}\right)^{\frac{p}{2}}\left(1+C_{1, p}\right)(t-s)^{\frac{p}{2}}=C_{2, p}(t-s)^{\frac{p}{2}},
\end{aligned}
$$

where

$$
\gamma=2^{p-1}(t-s)^{p-1} K^{\frac{p}{2}}+2^{p-1}\left(\frac{p(p-1)}{2}\right)^{\frac{p}{2}}(t-s)^{\frac{p-2}{2}} K^{\frac{p}{2}}
$$

and

$$
C_{2, p}=\frac{1}{2}(8 K)^{\frac{p}{2}}\left(\frac{p(p-1)}{2}\right)^{\frac{p}{2}}\left(1+C_{1, p}\right)
$$

Lemma 2.5 Under the linear growth condition (3), for all $n \geq 1$ and $1<$ $p<2$,

$$
\sup _{t_{0} \leq t \leq T} \mathbb{E}\left|x_{n}(t)\right|^{p} \leq \bar{C}_{1, p}
$$

Proof Note that the Hölder inequality implies

$$
\mathbb{E}\left|x_{n}(t)\right|^{p} \leq\left[\mathbb{E}\left|x_{n}(t)\right|^{2}\right]^{\frac{p}{2}}
$$

Thus, if $1<p<2$, we have

$$
\sup _{t_{0} \leq t \leq T} \mathbb{E}\left|x_{n}(t)\right|^{p} \leq \sup _{t_{0} \leq t \leq T}\left[\mathbb{E}\left|x_{n}(t)\right|^{2}\right]^{\frac{p}{2}} \leq\left(C_{1}\right)^{\frac{p}{2}}=\bar{C}_{1, p}
$$

Lemma 2.6 Under the linear growth condition (3), for all $n \geq 1$ and $t_{0} \leq$ $s<t \leq T$ with $t-s \leq 1$ and $1<p<2$,

$$
\mathbb{E}\left|x_{n}(t)-x_{n}(s)\right|^{p} \leq \bar{C}_{2, p}(t-s)^{\frac{p}{2}} .
$$


Proof Since $x_{n}(t)-x_{n}(s)=\int_{s}^{t} f\left(\hat{x}_{n}(r), r\right) d r+\int_{s}^{t} g\left(\hat{x}_{n}(r), r\right) d B(r)$, then we have

$$
\begin{aligned}
& \mathbb{E}\left|x_{n}(t)-x_{n}(s)\right|^{p} \\
\leq & {[2(t-s)]^{p-1} \mathbb{E} \int_{s}^{t}\left|f\left(\hat{x}_{n}(r), r\right)\right|^{p} d r+2^{p-1}\left[\mathbb{E} \int_{s}^{t}\left|g\left(\hat{x}_{n}(r), r\right)\right|^{2} d r\right]^{\frac{p}{2}} } \\
\leq & {[2(t-s)]^{p-1} K^{\frac{p}{2}} \int_{s}^{t}\left(1+\mathbb{E} \sup _{s \leq u \leq r}\left|x_{n}(u)\right|^{p}\right) d r+2^{p-1} K^{\frac{p}{2}}\left(\int_{s}^{t}\left(1+\mathbb{E} \sup _{s \leq u \leq r}\left|x_{n}(u)\right|^{2}\right) d r\right)^{\frac{p}{2}} } \\
\leq & 2^{p-1} K^{\frac{p}{2}}(t-s)^{p}\left(1+\bar{C}_{1, p}\right)+2^{p-1} K^{\frac{p}{2}}(t-s)^{\frac{p}{2}}\left(1+C_{1}\right)^{\frac{p}{2}} \\
\leq & 2^{p-1} K^{\frac{p}{2}}\left[1+\bar{C}_{1, p}+\left(1+C_{1}\right)^{\frac{p}{2}}\right](t-s)^{\frac{p}{2}}=\bar{C}_{2, p}(t-s)^{\frac{p}{2}} .
\end{aligned}
$$

Hence, the inequality (16) can be obtained.

\subsection{Proofs of main results}

In this subsection, we start to prove our main theorems.

Proof [Proof of Theorem 2.1] According to the expressions of $x_{n}(t)$ and $x(t)$, we have

$$
\begin{aligned}
& \left|x_{n}(t)-x(t)\right|^{p} \\
= & \left|\int_{t_{0}}^{t}\left[f\left(\hat{x}_{n}(s), s\right)-f(x(s), s)\right] d s+\int_{t_{0}}^{t}\left[g\left(\hat{x}_{n}(s), s\right)-g(x(s), s)\right] d B(s)\right|^{p} \\
\leq & 2^{p-1}\left|\int_{t_{0}}^{t}\left[f\left(\hat{x}_{n}(s), s\right)-f(x(s), s)\right] d s\right|^{p}+2^{p-1}\left|\int_{t_{0}}^{t}\left[g\left(\hat{x}_{n}(s), s\right)-g(x(s), s)\right] d B(s)\right|^{p}
\end{aligned}
$$

Thus, it is not difficult to derive that

$$
\begin{aligned}
& \mathbb{E}\left(\sup _{t_{0} \leq t \leq T}\left|x_{n}(t)-x(t)\right|^{p}\right) \\
\leq & 2^{p-1}\left(T-t_{0}\right)^{p-1} \bar{K}^{\frac{p}{2}} \int_{t_{0}}^{t} \mathbb{E}\left|\hat{x}_{n}(s)-x(s)\right|^{p} d s \\
+ & 2^{p-1}\left(\frac{p^{3}}{2(p-1)}\right)^{\frac{p}{2}}\left(T-t_{0}\right)^{\frac{p-2}{2}} \bar{K}^{\frac{p}{2}} \int_{t_{0}}^{t} \mathbb{E}\left|\hat{x}_{n}(s)-x(s)\right|^{p} d s \\
\leq & 2^{p-1} M \int_{t_{0}}^{t} \mathbb{E}\left|\hat{x}_{n}(s)-x_{n}(s)\right|^{p} d s+2^{p-1} M \int_{t_{0}}^{t} \mathbb{E}\left|x_{n}(s)-x(s)\right|^{p} d s,
\end{aligned}
$$

where $M=2^{p-1}\left(T-t_{0}\right)^{\frac{p-2}{2}} \bar{K}^{\frac{p}{2}}\left[\left(T-t_{0}\right)^{\frac{p}{2}}+\left(\frac{p^{3}}{2(p-1)}\right)^{\frac{p}{2}}\right]$. 
However, by Lemma 2.4, we have the following fact:

$$
\begin{aligned}
& \int_{t_{0}}^{t} \mathbb{E}\left|\hat{x}_{n}(s)-x_{n}(s)\right|^{p} d s \\
= & \int_{t_{0}}^{t_{0}+\frac{1}{n}} \mathbb{E}\left|x_{n}\left(t_{0}\right)-x_{n}(s)\right|^{p} d s+\int_{t_{0}+\frac{1}{n}}^{t_{0}+\frac{2}{n}} \mathbb{E}\left|x_{n}\left(t_{0}+\frac{1}{n}\right)-x_{n}(s)\right|^{p} d s+\ldots \\
\leq & \frac{C_{2, p}}{n^{\frac{p}{2}}} \cdot \frac{1}{n} \cdot \frac{t-t_{0}}{\frac{1}{n}} \leq \frac{C_{2, p}\left(T-t_{0}\right)}{n^{\frac{p}{2}}} .
\end{aligned}
$$

Therefore, it follows from the upper inequality that

$$
\begin{aligned}
& \mathbb{E}\left(\sup _{t_{0} \leq t \leq T}\left|x_{n}(t)-x(t)\right|^{p}\right) \\
\leq & 2^{p-1} M \frac{C_{2, p}\left(T-t_{0}\right)}{n^{\frac{p}{2}}}+2^{p-1} M \int_{t_{0}}^{t} \mathbb{E}\left(\sup _{t_{0} \leq r \leq s}\left|x_{n}(r)-x(r)\right|^{p}\right) d s .
\end{aligned}
$$

Finally, according to the Gronwall inequality, we will have

$$
\begin{aligned}
& \mathbb{E}\left(\sup _{t_{0} \leq t \leq T}\left|x_{n}(t)-x(t)\right|^{p}\right) \\
\leq & 2^{p-1} M \frac{C_{2, p}\left(T-t_{0}\right)}{n^{\frac{p}{2}}} e^{2^{p-1} M\left(T-t_{0}\right)}=\frac{C_{3, p}}{n^{\frac{p}{2}}} .
\end{aligned}
$$

Then, the required inequality (11) can be obtained.

Proof [Proof of Theorem 2.2] As the similar as the proof of Theorem 2.1, we can still have the following inequality:

$$
\begin{aligned}
\left|x_{n}(t)-x(t)\right|^{p} & \leq 2^{p-1}\left|\int_{t_{0}}^{t}\left[f\left(\hat{x}_{n}(s), s\right)-f(x(s), s)\right] d s\right|^{p} \\
& +2^{p-1}\left|\int_{t_{0}}^{t}\left[g\left(\hat{x}_{n}(s), s\right)-g(x(s), s)\right] d B(s)\right|^{p} .
\end{aligned}
$$

Hence, by using the Hölder inequality and the Burkholder-Davis-Gundy inequality, we have

$$
\begin{aligned}
\mathbb{E}\left(\sup _{t_{0} \leq t \leq T}\left|x_{n}(t)-x(t)\right|^{p}\right) \leq & 2^{p-1}\left(T-t_{0}\right)^{p-1} \mathbb{E} \int_{t_{0}}^{T}\left|f\left(\hat{x}_{n}(s), s\right)-f(x(s), s)\right|^{p} d s \\
& +2^{p-1}\left(\frac{32}{p}\right)^{\frac{p}{2}}\left[\mathbb{E} \int_{t_{0}}^{T}\left|g\left(\hat{x}_{n}(s), s\right)-g(x(s), s)\right|^{2} d s\right]^{\frac{p}{2}} .
\end{aligned}
$$


From Theorem 1.1 and some conclusions which have proved in the upper contents, we have

$$
\begin{aligned}
& 2^{p-1}\left(\frac{32}{p}\right)^{\frac{p}{2}}\left[\mathbb{E} \int_{t_{0}}^{T}\left|g\left(\hat{x}_{n}(s), s\right)-g(x(s), s)\right|^{2} d s\right]^{\frac{p}{2}} \\
\leq & 2^{\frac{3}{2} p-1} \bar{K}^{\frac{p}{2}}\left(\frac{32}{p}\right)^{\frac{p}{2}}\left[\left(\int_{t_{0}}^{T} \mathbb{E}\left|\hat{x}_{n}(s)-x_{n}(s)\right|^{2} d s\right)^{\frac{p}{2}}+\left(\int_{t_{0}}^{T} \mathbb{E}\left|x_{n}(s)-x(s)\right|^{2} d s\right)^{\frac{p}{2}}\right] \\
\leq & 2^{\frac{3}{2} p-1} \bar{K}^{\frac{p}{2}}\left(\frac{32}{p}\right)^{\frac{p}{2}}\left(T-t_{0}\right)^{\frac{p}{2}}\left[\left(C_{2}\right)^{\frac{p}{2}}+\left(C_{3}\right)^{\frac{p}{2}}\right]\left(\frac{1}{n}\right)^{\frac{p}{2}}
\end{aligned}
$$

Furthermore, by Lemma 2.6, we can get

$$
\begin{aligned}
& 2^{p-1}\left(T-t_{0}\right)^{p-1} \mathbb{E} \int_{t_{0}}^{T}\left|f\left(\hat{x}_{n}(s), s\right)-f(x(s), s)\right|^{p} d s \\
& \leq 2^{2 p-2}\left(T-t_{0}\right)^{p-1} \bar{K}^{\frac{p}{2}} \int_{t_{0}}^{T} \mathbb{E}\left|\hat{x}_{n}(s)-x_{n}(s)\right|^{p} d s \\
& \quad+2^{2 p-2}\left(T-t_{0}\right)^{p-1} \bar{K}^{\frac{p}{2}} \int_{t_{0}}^{T} \mathbb{E}\left|x_{n}(s)-x(s)\right|^{p} d s \\
& \leq 2^{2 p-2}\left(T-t_{0}\right)^{p-1} \bar{K}^{\frac{p}{2}} \int_{t_{0}}^{T} \mathbb{E}\left(\sup _{t_{0} \leq r \leq s}\left|x_{n}(r)-x(r)\right|^{p}\right) d s \\
& +2^{2 p-2}\left(T-t_{0}\right)^{p} \bar{K}^{\frac{p}{2}}\left(\frac{1}{n}\right)^{\frac{p}{2}} \bar{C}_{2, p}
\end{aligned}
$$

Thus, we have

$$
\begin{aligned}
& \mathbb{E}\left(\sup _{t_{0} \leq t \leq T}\left|x_{n}(t)-x(t)\right|^{p}\right) \\
\leq & 2^{\frac{3}{2} p-1} \bar{K}^{\frac{p}{2}}\left(\frac{32}{p}\right)^{\frac{p}{2}}\left(T-t_{0}\right)^{\frac{p}{2}}\left(\frac{1}{n}\right)^{\frac{p}{2}}\left[\left(C_{2}\right)^{\frac{p}{2}}+\left(C_{3}\right)^{\frac{p}{2}}\right]+2^{2 p-2}\left(T-t_{0}\right)^{p} \bar{K}^{\frac{p}{2}}\left(\frac{1}{n}\right)^{\frac{p}{2}} \bar{C}_{2, p} \\
& +2^{2 p-2}\left(T-t_{0}\right)^{p-1} \bar{K}^{\frac{p}{2}} \int_{t_{0}}^{T} \mathbb{E}\left(\sup _{t_{0} \leq r \leq s}\left|x_{n}(r)-x(r)\right|^{p}\right) d s \\
\leq & \left(\frac{1}{n}\right)^{\frac{p}{2}} 2^{2 p-1} \bar{K}^{\frac{p}{2}}\left(T-t_{0}\right)^{\frac{p}{2}}\left[\left(\frac{16 C_{2}}{p}\right)^{\frac{p}{2}}+\left(\frac{16 C_{3}}{p}\right)^{\frac{p}{2}}+\frac{\bar{C}_{2, p}}{2}\left(T-t_{0}\right)^{\frac{p}{2}}\right] \\
& +2^{2 p-2}\left(T-t_{0}\right)^{p-1} \bar{K}^{\frac{p}{2}} \int_{t_{0}}^{T} \mathbb{E}\left(\sup _{t_{0} \leq r \leq s}\left|x_{n}(r)-x(r)\right|^{p}\right) d s .
\end{aligned}
$$

Finally, the desired inequality (12) can be obtained by applying the Gronwall inequality. 


\section{References}

[1] X. R. Mao, Approximate solutions for stochastic differential equations with pathwise uniqueness. Stochastic Anal. Appl. 12 (1994), no. 3, 355367.

[2] X. R. Mao, Stochastic differential equations and their applications. Horwood Publishing Limited, Chichester, 1997.

Received: June 1, 2014 